\title{
Enhanced thermoelectric properties in hybrid graphene/boron nitride nanoribbons
}

\author{
Kaike Yang, ${ }^{1,2}$ Yuanping Chen, ${ }^{1, *}$ Roberto D’Agosta,,${ }^{2,3, \dagger}$ Yuee Xie, ${ }^{1}$ Jianxin Zhong, ${ }^{1}$ and Angel Rubio ${ }^{2, \ddagger}$ \\ ${ }^{1}$ Laboratory for Quantum Engineering and Micro-Nano Energy Technology and Department of Physics, Xiangtan University, \\ Xiangtan 411105, Hunan, China \\ ${ }^{2}$ Nano-Bio Spectroscopy Group and ETSF Scientific Development Centre, Departamento Fisica de Materiales, Universidad del Pais Vasco \\ UPVIEHU, Centro de Fisica de Materiales CSIC-UPVIEHU and DIPC, Avenida Tolosa 72, E-20018 San Sebastian, Spain
}

${ }^{3}$ IKERBASQUE, Basque Foundation for Science, E-48011, Bilbao, Spain

(Received 5 April 2012; published 16 July 2012)

\begin{abstract}
The thermoelectric properties of hybrid graphene/boron nitride nanoribbons (BCNNRs) are investigated using the nonequilibrium Green's function approach. We find that the thermoelectric figure of merit $(Z T)$ can be remarkably enhanced by periodically embedding hexagonal $\mathrm{BN}(h-\mathrm{BN})$ into graphene nanoribbons (GNRs). Compared to pristine GNRs, the $Z T$ for armchair-edged BCNNRs with width index $3 p+2$ is enhanced 10-20 times, while the $Z T$ of nanoribbons with other widths is enhanced by just 1.5-3 times. As for zigzag-edge nanoribbons, the $Z T$ is enhanced 2-3 times. This improvement comes from the combined increase in the Seebeck coefficient and the reduction in the thermal conductance outweighing the decrease in the electrical conductance. In addition, the effect of the component ratio of $h$-BN on the thermoelectric transport properties is discussed. These results qualify BCNNRs as a promising candidate for building outstanding thermoelectric devices.
\end{abstract}

DOI: 10.1103/PhysRevB.86.045425

PACS number(s): 68.65.Pq, 73.50.Lw

\section{INTRODUCTION}

Thermoelectric materials that can convert heat into electric energy have attracted increasing attention from both theoretical and experimental research due to their potential technological applications. ${ }^{1-5}$ The thermoelectric conversion efficiency is measured by the figure of merit ${ }^{6-9}$

$$
Z T=\frac{\sigma S^{2} T}{\left(\kappa_{e}+\kappa_{p}\right)},
$$

where $\sigma, S, T, \kappa_{e}$, and $\kappa_{p}$ represent the electrical conductance, Seebeck coefficient, absolute temperature, and the electron and phonon contributions to thermal conductance, respectively. Clearly, an optimal thermoelectric material should bear high electrical conductance, high Seebeck coefficient, and correspondingly, low thermal conductance.

Graphene and hexagonal boron nitride $(h$-BN) are singleatomic-layer honeycomb materials. ${ }^{10-18}$ Because of the quantum confinement effect, graphene and $h$-BN exhibit very high electrical conductance and Seebeck coefficient. ${ }^{19-21}$ However, both pristine graphene and pristine $h$-BN are poor thermoelectric materials because their thermal conductance is also very high. ${ }^{22-25}$ Recently, a novel nanomaterial compounded of graphene and $h$-BN has been synthesized by the thermal catalytic chemical vapor deposition method. ${ }^{26}$ This novel hybrid nanostructure possesses physical properties distinct from both pristine graphene and pristine $h$-BN. ${ }^{27-40}$ For example, a tunable electronic energy gap dependent on the concentration of $h$-BN emerges in the two-dimensional hybrid sheet; ${ }^{41,42}$ while in the one-dimensional hybrid nanoribbons, it is found that the armchair-edged graphene nanoribbons ( $A$-GNRs) embedded in $h$-BN are always semiconducting while zigzag-edged structures become half-metallic; ${ }^{43,44}$ the thermal conductance of hybrid nanoribbons is obviously reduced by the interface scattering. ${ }^{45}$ Previous studies only focused on electronic and thermal transport, ${ }^{43-45}$ respectively, while the thermoelectric properties of hybrid graphene and $h$-BN (BCN) have been left unexplored thus far. Because ordered superlattices can form promising structures for improving thermoelectric performance, ${ }^{46}$ it is natural to look into what happens if we construct a periodic structure composed of alternating $h$-BN and graphene.

In this paper, we investigate the thermoelectric properties of BCN nanoribbons (BCNNRs) by using the nonequilibrium Green's function (NEGF) approach. ${ }^{4-52}$ It is found that the figure of merit can be remarkably enhanced by periodically embedding $h$-BN into the GNRs. In particular, the $Z T$ is enhanced 10-20 times for metallic $A$-GNRs with width index $3 p+2$ (where $p$ is a positive integer), while the $Z T$ of nanoribbons with other widths is enhanced $\sim 1.5-3$ times. Regarding the zigzag-edge structure, the figure of merit for BCNNRs is improved 2-3 times compared to that of pristine zigzag-edged GNRs ( $Z$-GNRs). The origin of this enhancement effect is analyzed: it is found that both the electrical conductance and thermal conductance decrease, whereas the Seebeck coefficient increases with the periodic number, $N$. In addition, the influence of the component ratio of $h$-BN on the thermoelectric transport properties is also discussed. Here, it is shown that the figure of merit of BCNNRs at component ratio near 0.5 assumes the largest value. These results indicate that the BCNNRs could be a very promising candidate for the design of excellent thermoelectric devices.

\section{MODEL AND METHOD}

Let us consider the armchair-edged BCNNRs ( $A$-BCNNRs) shown in Figs. 1(a) and 1(b) and zigzag-edged BCNNRs (Z-BCNNRs) shown in Figs. 1(c) and 1(d), respectively. The systems are divided into three parts. The left and right leads composed of GNRs have perfect periodicity along the ribbon axis and the ribbon widths are labeled by $W_{A}$ and $W_{Z}$ for armchair and zigzag edges, respectively. The central scattering region is the hybridized structure consisting of alternating $h$-BN and graphene supercells. $L_{S}=\left(L_{B N}+L_{C}\right)$ and $L_{S}^{\prime}=\left(L_{B N}^{\prime}+L_{C}^{\prime}\right)$ are the lengths of the supercell in armchair- and zigzag-edged nanoribbons, respectively, where 
Left lead Central scattering region

Right lead

(a)

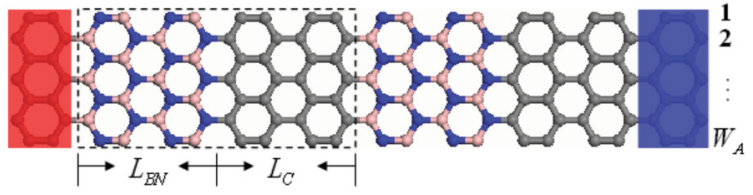

(b)

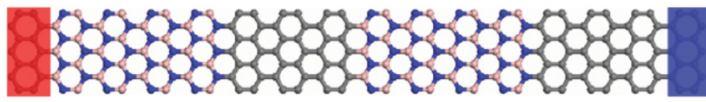

(c)

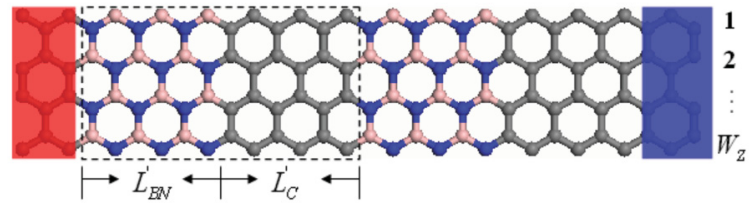

(d)

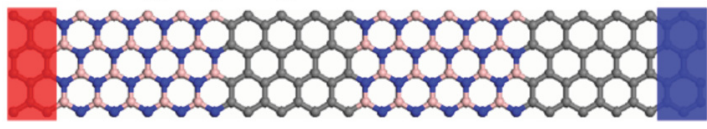

FIG. 1. (Color online) Schematics of (a) and (b) A-BCNNRs, and (c) and (d) Z-BCNNRs. The left and right leads made of GNRs have perfect periodicity along the ribbon axis. The central scattering region is formed by periodic alternating of $h$-BN and graphene. The dotted pane denotes a supercell in which $L_{B N}\left(L_{B N}^{\prime}\right)$ and $L_{C}\left(L_{C}^{\prime}\right)$ separately represent the lengths of $h$-BN and graphene for armchairand zigzag-edged nanoribbons in the longitudinal direction, while $W_{A}$ and $W_{Z}$ are used to denote the width of the nanoribbons, respectively. Note that in (a) and (c) the length of $h$-BN equals the length of graphene, while in (b) and (d) they are different.

$L_{B N}\left(L_{B N}^{\prime}\right)$ and $L_{C}\left(L_{C}^{\prime}\right)$ separately represent the lengths of $h$-BN and graphene in the supercell in the longitudinal direction. We want to point out that in Figs. 1(a) and 1(c) the length of $h$-BN equals the length of graphene, while their lengths in Figs. 1(b) and 1(d) are different in the supercell. We use $N$ for the number of supercells and the component ratio is defined as $\gamma=L_{B N} / L_{S}$ in $A$-BCNNRs and $\gamma^{\prime}=L_{B N}^{\prime} / L_{S}^{\prime}$ in $Z$-BCNNRs. To investigate the thermoelectric properties of these structures, the NEGF method is employed. For the BCNNRs, the tight-binding Hamiltonian for electrons is written as

$$
\begin{aligned}
H= & \sum_{\alpha=L, C, R} H_{\alpha}+\sum_{\beta=L, R}\left(H^{\beta C}+H^{C \beta}\right) \\
= & \sum_{\alpha=L, C, R} H_{\alpha}+\sum_{i, j} t_{i, j}^{L C}\left(a_{i}^{\dagger L} a_{j}^{C}+\text { H.c. }\right) \\
& +\sum_{i, j} t_{i, j}^{R C}\left(a_{i}^{\dagger R} a_{j}^{C}+\text { H.c. }\right)
\end{aligned}
$$

where $H_{\alpha}=\sum_{i} \varepsilon_{i}^{\alpha} a_{i}^{\dagger \alpha} a_{i}^{\alpha}+\sum_{i, j} t_{i, j}^{\alpha} a_{i}^{\dagger \alpha} a_{j}^{\alpha}, a_{i}^{\dagger \alpha}$ and $a_{i}^{\alpha}$ are the creation and annihilation operators, $\varepsilon_{i}^{\alpha}$ is the site energy at the $i$ th atom, and $t_{i, j}^{\alpha}$ is the hopping integral for nearest neighbors, respectively. We take the parameters of the Hamiltonian from Ref. 53 for graphene and Ref. 54 for $h$-BN, and take the average value for the hopping energy between carbon and boron or nitrogen atoms. The tight-binding parameters correspond to the $\pi-\pi^{*}$ chemical bonds. In general for $h$-BN, we should also consider the contribution of the $\sigma-\sigma^{*}$ bonds. However, as we will show in the following for calculating the figure of merit, the contribution of those bands can be
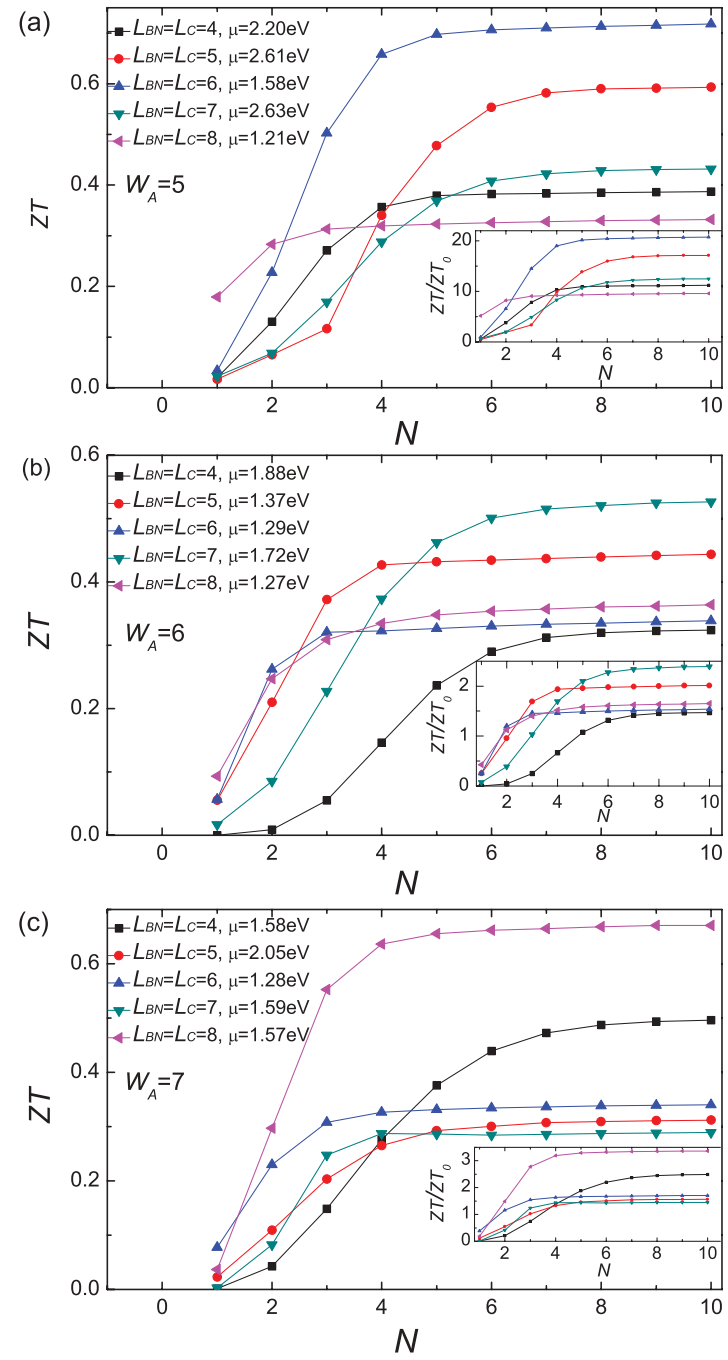

FIG. 2. (Color online) Thermoelectric figure of merit $Z T$ for $A$-BCNNRs with different widths: (a) $W_{A}=5$, (b) $W_{A}=6$, and (c) $W_{A}=7$, while the insets plot the ratio $Z T / Z T_{0}$ as a function of periodic number $N$ for the nanoribbons, where $Z T_{0}$ corresponds to the result of perfect $A$-GNRs. The temperature $T=300 \mathrm{~K}$ and the component ratio $\gamma=0.5$ (i.e., $L_{B N}=L_{C}$ ). For given lengths $L_{B N}$ and $L_{C}$, we take the chemical potential $(\mu)$ corresponding to the absolute maximum of the figure of merit (a detailed discussion is given in the main text).

safely neglected. Starting from this Hamiltonian, the retarded Green's function is expressed as

$$
G^{r}(E)=\left[\left(E+i 0^{+}\right) I-H_{C}-\sum_{L}^{r}-\sum_{R}^{r}\right]^{-1},
$$

where $E$ is the electron energy, $I$ is the identity matrix, $H_{C}$ is the Hamiltonian of the central scattering region, $\sum_{\beta}^{r}=H^{C \beta} g_{\beta}^{r} H^{\beta C}$ denotes the self-energy of the left $(L)$ and right $(R)$ regions, and $g_{\beta}^{r}$ is the surface Green's function of the leads which can be computed by a recursive iteration technique. ${ }^{55}$ Once the retarded Green's function is obtained, we can calculate the electronic transmission coefficient

$$
T_{e}(E)=\operatorname{Tr}\left[G^{r}(E) \Gamma_{L} G^{a}(E) \Gamma_{R}\right],
$$



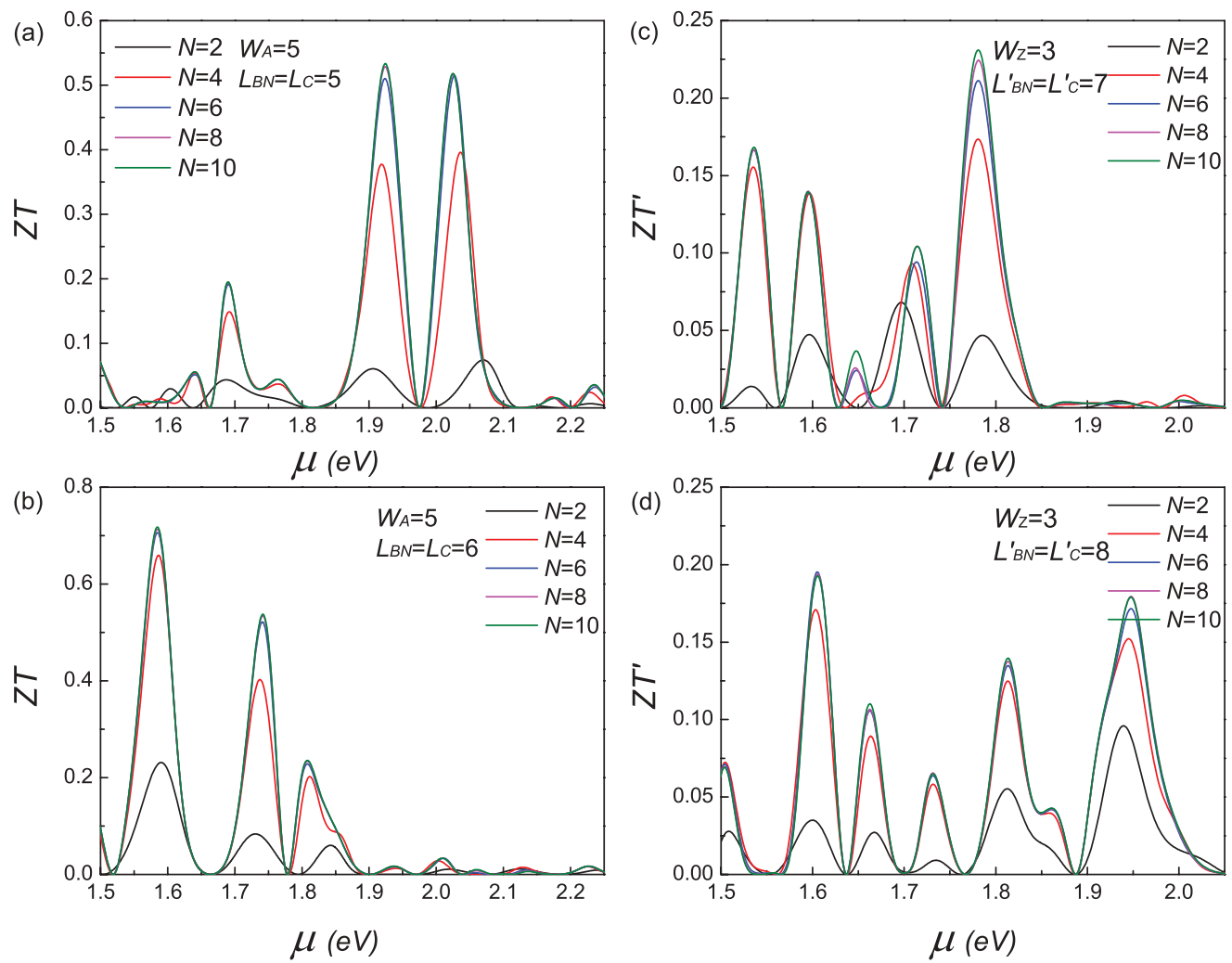

FIG. 3. (Color online) (a) and (b) Thermoelectric figure of merit $(Z T)$ as a function of chemical potential $(\mu)$ for $A$-BCNNRs with width $W_{A}=5$ at different periodic number $(N)$ and different length of $h$-BN and graphene nanoribbons: (a) $L_{B N}=L_{C}=5$ and (b) $L_{B N}=L_{C}=6$. (c) and (d) Thermoelectric figure of merit $\left(Z T^{\prime}\right)$ as a function of chemical potential $(\mu)$ for $Z$-BCNNRs with width $W_{Z}=3$ at different periodic number $(N)$ and different length of $h$-BN and graphene nanoribbons: (c) $L_{B N}^{\prime}=L_{C}^{\prime}=7$ and (d) $L_{B N}^{\prime}=L_{C}^{\prime}=8$. To evaluate the figure of merit, in all cases we have fixed the chemical potential by taking the absolute maximum of $Z T$ as a function of the periodic number and length of the graphene and BN nanoribbons. The temperature is $T=300 \mathrm{~K}$.

where $G^{a}(\omega)=\left[G^{r}(\omega)\right]^{\dagger}$ is the advanced Green's function, and $\Gamma_{\beta}=i\left(\sum_{\beta}^{r}-\sum_{\beta}^{a}\right)$ describes the interaction between the left or right lead and the central region. By using the transmission coefficient, the electrical conductance, the Seebeck coefficient, and the electron contributed thermal conductance can be calculated in linear response separately from

$$
\begin{aligned}
\sigma(\mu) & =e^{2} L_{0}(\mu, T), \\
S(\mu) & =\frac{1}{e T} \frac{L_{1}(\mu, T)}{L_{0}(\mu, T)}, \\
\kappa_{e}(\mu) & =\frac{1}{T}\left[L_{2}(\mu, T)-\frac{L_{1}(\mu, T)^{2}}{L_{0}(\mu, T)}\right],
\end{aligned}
$$

where $\quad L_{n}(\mu, T)=(2 / h) \int_{-\infty}^{\infty} d E T_{e}(E)(E-\mu)^{n}\left[-\left(\partial f_{e}\right.\right.$ $(E, \mu, T) / \partial E)]$ is the Lorenz function, $\mu$ is the chemical potential, $e$ is the electric charge of carriers, $f_{e}$ is the Fermi-Dirac distribution function at temperature $T$, and $h$ is the Planck constant. From the expression of the Lorenz function, it now becomes clear why we can neglect the contribution of the $\sigma$ bands. At finite temperature, the derivative of the Fermi function effectively restricts the integration range to a small region close to the chemical potential. Normally, the chemical potential is small compared with the energy between the $\sigma$ and $\pi$ bands in $h$-BN.

On the other hand, for thermal transport, according to Refs. 48-50, the phonon-transmission coefficient $T_{p}(\omega)$ can be calculated in the harmonic approximation with the same steps as for the electrons. Indeed, in Eq. (3) one only needs to change $E$ into $\omega^{2}, H_{C}$ into $K_{C}$, and correspondingly compute the self-energy, and then get the phonon retarded Green's function, where $\omega$ is the vibrational frequency of phonons and $K_{C}$ is the spring constant matrix of the central region ${ }^{24,25,50,56-58}$ (the parameters are taken from Ref. 57 for graphene and Ref. 58 for $h$-BN). We take the average value for the force constant between graphene and $h$-BN. The presence of the square of the frequency, $\omega^{2}$, instead of the phonon energy stems from the different analytical properties of the phonon Green's functions. Finally, the phonons contributed thermal conductance is given by

$$
\kappa_{p}(T)=\frac{h}{4 \pi^{2}} \int_{0}^{\infty} \frac{\partial f_{p}(\omega)}{\partial T} T_{p}(\omega) \omega d \omega,
$$

where $f_{p}(\omega)$ is the Bose-Einstein distribution function for heat carriers. We have therefore all the ingredients to calculate the figure of merit.

\section{RESULTS AND DISCUSSION}

In Fig. 2, we investigate the thermoelectric transport properties of $A$-BCNNRs with different widths as a function of the number of supercell $N$ for $\gamma=0.5\left(L_{B N}=L_{C}\right)$. Figure 2(a) shows $Z T$ for $A$-BCNNRs with width $W_{A}=5$, and the inset plots the ratio $Z T / Z T_{0}$, where $Z T_{0}$ is the figure of merit of 

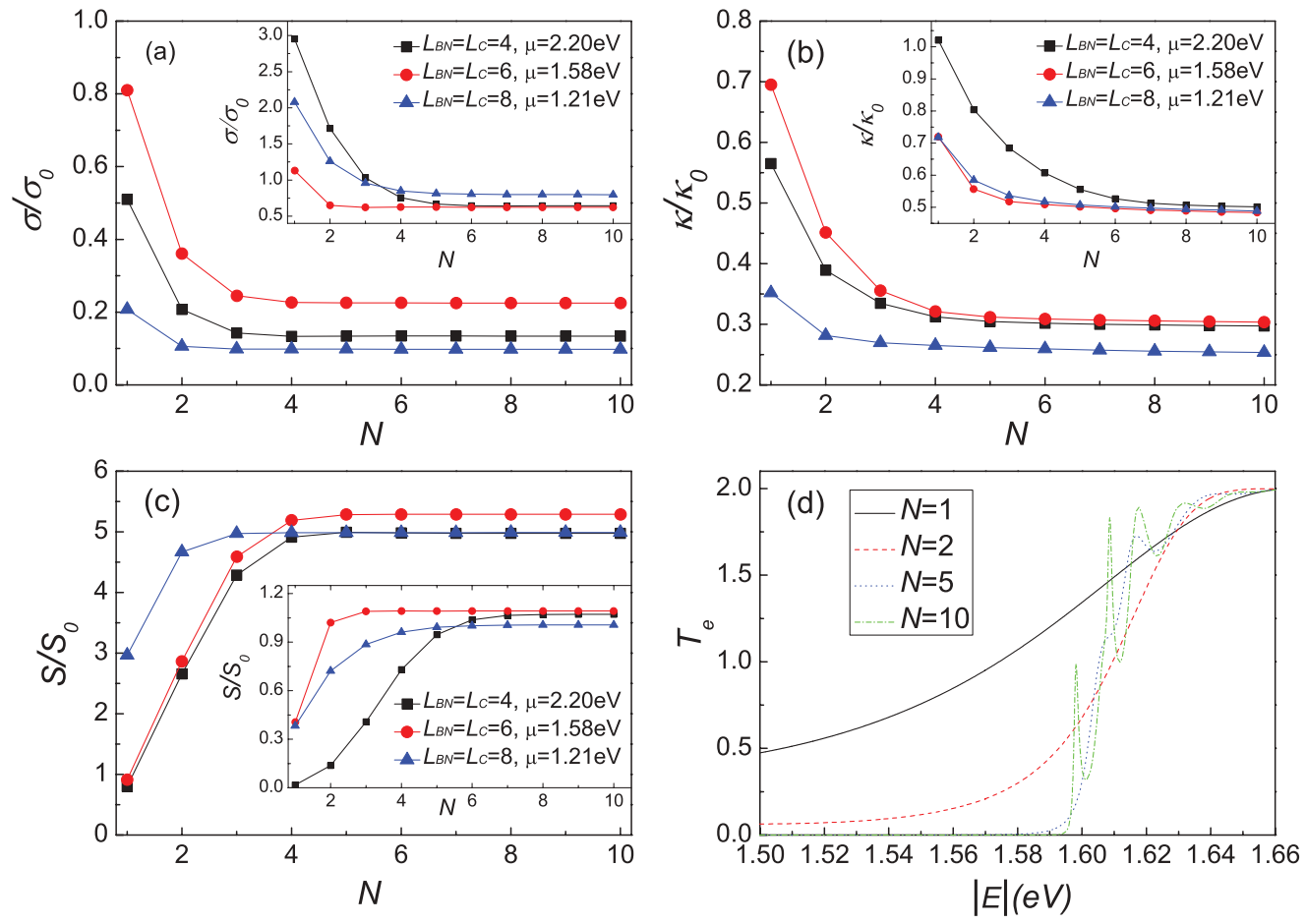

FIG. 4. (Color online) (a) Electrical conductance ratio $\sigma / \sigma_{0}$, (b) thermal conductance ratio $\kappa / \kappa_{0}$, and (c) Seebeck coefficient ratio $S / S_{0}$ as a function of periodic number $N$ for $A$-BCNNRs with width $W_{A}=5$, where $\sigma_{0}, \kappa_{0}$, and $S_{0}$ correspond to pristine $A$-GNRs; the insets show the results of nanoribbons with width $W_{A}=6$. (d) Transmission coefficient $T_{e}$ versus electronic energy $E$ for $A$-BCNNRs with $W_{A}=5$ at $L_{B N}=L_{C}=6$ and $\mu=1.58 \mathrm{eV}$. The other parameters are the same as in Fig. 2.

pristine $A$-GNRs. In Fig. 2, the figure of merit plotted as a function of the geometrical properties of the nanoribbons for different lengths of the device are obtained by taking the maximum $Z T$ as a function of the average chemical potential of the leads, where the chemical potential is taken to be small enough that the influence of interface states can be neglected. To do so, we have calculated $Z T$ as a function of the chemical potential for all the geometries, and taken the absolute maximum of the figure of merit (see Fig. 3). For given lengths $L_{B N}$ and $L_{C}$, we fix the chemical potential to the value that provides this absolute maximum also a function of the length $N$. This procedure fixes the chemical potential of the nanoribbon. We then use this value of the chemical potential to evaluate the figure of merit. We would like to point out that this procedure gives the maximum value of $Z T\left(Z T^{\prime}\right)$ for almost any $N$, as seen from Fig. 3. Only for small $N$, better values of $Z T\left(Z T^{\prime}\right)$ could be obtained by considering a different chemical potential.

One can find from Fig. 2(a) that when the periodic number $N=1, Z T$ is relatively small. Increasing $N, Z T$ gradually increases for various values of $L_{B N}$ and $L_{C}$. However, the magnitude of the enhancement is different, as can be seen from the inset of Fig. 2(a). At $L_{B N}=L_{C}=4$, 7, and 8, it is found that the figure of merit for $A$-BCNNRs is enhanced about ten times compared to that of pristine metallic $A$-GNRs. Interestingly, in the case of $L_{B N}=L_{C}=5$ and 6 , a quite higher value $Z T / Z T_{0} \approx 15-20$ is attained. When the periodic number is greater than 6 , we find that $Z T / Z T_{0}$ approaches almost a constant value. The reason for this behavior is easily explained: when the periodic number is sufficiently large, the whole nanoribbons can be approximately regarded as a superlattice structure composed of alternating $h$-BN and graphene; then the transmission of electron and phonon in the $A$-BCNNRs is equivalent to that of the superlattice.

In Figs. 2(b) and 2(c), the thermoelectric properties of semiconducting $A$-GNRs embedded with $h$-BN are investigated. As a comparison, we plot the figure of merit for $A$-BCNNRs with width $W_{A}=6$ and 7 . It can be seen that similarly to the case of metallic nanoribbons with width $W_{A}=5, Z T$ increases with the periodic number $N$ and finally approaches a constant value for different $L_{B N}$ and $L_{C}$. Nevertheless, compared to the perfect semiconducting $A$-GNRs, it is found that the figure of merit for $A$-BCNNRs is about 1.5-3.0 times larger than that of pristine graphene nanoribbons (see the insets). With further increasing the ribbon width, $Z T$ is also increased with the periodic number and the enhanced magnitude is different for metallic and semiconducting $A$-GNRs. The $Z T$ of metallic $A$-GNRs is enhanced 10-20 times, while the $Z T$ of semiconducting $A$-GNRs is enhanced about 1.5-3 times.

To elucidate the origin of the thermoelectric figure of merit enhancement in A-BCNNRs, Figs. 4(a)-4(c) respectively depict the electrical conductance ratio $\left(\sigma / \sigma_{0}\right)$, thermal conductance ratio $\left(\kappa / \kappa_{0}\right)$, and Seebeck coefficient ratio $\left(S / S_{0}\right)$ as a function of the periodic number $N$ for the ribbon width $W_{A}=5$, and accordingly, in the insets we plot the same quantities for nanoribbons with $W_{A}=6$. The quantities with subscript 0 correspond to the parameters of pristine $A$-GNRs. It can be seen that both the electrical conductance and thermal conductance ratios decrease with increasing the periodic number, although the magnitude of the attenuation is different. In contrast to the perfect $A$-GNRs, the electrical conductance of $A$-BCNNRs is decreased nearly 2-3 times, while the thermal conductance is decreased 1-2 times, which suggests 

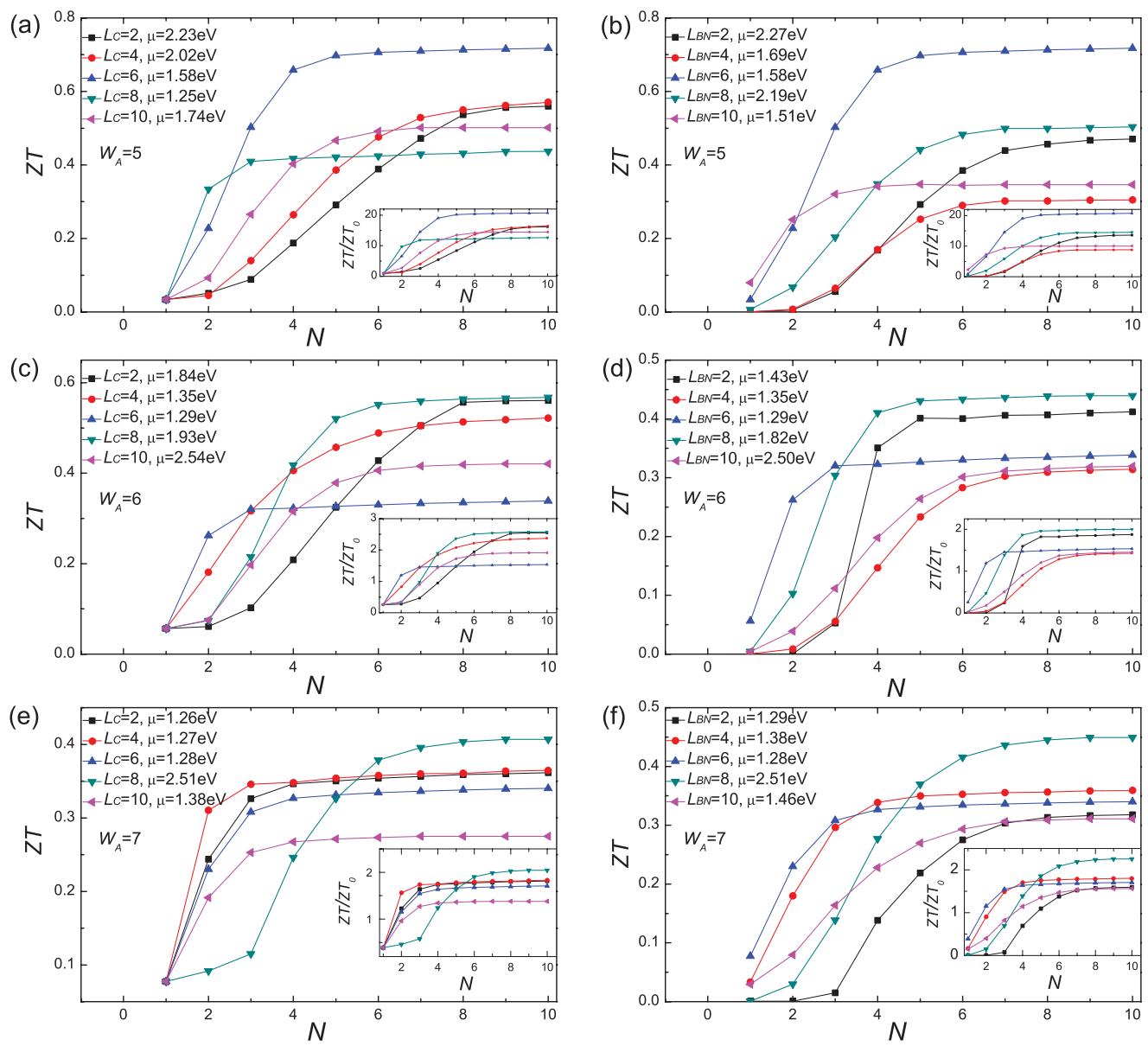

FIG. 5. (Color online) Thermoelectric figure of merit $Z T$ for $A$-BCNNRs as a function of periodic number $N$. In the left column [(a), (c), and (e)] $L_{B N}=6$, while in the right column [(b), (d), and (f)] $L_{C}=6$. The insets plot the ratio $Z T / Z T_{0}$ of the nanoribbons. The chemical potential $(\mu)$ we have taken for different value $L_{B N}\left(L_{C}\right)$ corresponds to the absolute maximum of the figure of merit and the other parameters are set the same as in Fig. 2.

that the hybrid structure has strong effects on the electronic conduction. The decreased electrical and thermal conductance ratios can be attributed to the destructive channels which block the carrier transmission. From Fig. 4(c), it is seen that the ratio of Seebeck coefficients increases with the periodic number $N$. The magnitude of the Seebeck coefficient for $A$-BCNNRs with width $W_{A}=5$ is about five times larger than the Seebeck coefficient for pristine $A$-GNRs, and in any case larger than that of the nanoribbons with width $W_{A}=6$ [see the inset of Fig. 4(c)]. Therefore, the enhancement of the figure of merit is mainly due to the increase of the Seebeck coefficient and the corresponding decrease of the thermal conductance. Together, they outweigh the decrease in the electrical conductance. From Eq. (5b), one can find that the Seebeck coefficient is dependent on the ratio of Lorenz function $L_{1} / L_{0}$ :

$$
\begin{aligned}
L_{0}(\mu, T)= & \frac{2}{h} \int_{-\infty}^{\infty}\left[-\frac{\partial f_{e}(E, \mu, T)}{\partial E}\right] T_{e}(E) d E, \\
L_{1}(\mu, T)= & \frac{2}{h}\left\{\int_{-\infty}^{E<\mu}\left[-\frac{\partial f_{e}(E, \mu, T)}{\partial E}\right](E-\mu) T_{e}(E) d E\right. \\
& \left.+\int_{E>\mu}^{\infty}\left[-\frac{\partial f_{e}(E, \mu, T)}{\partial E}\right](E-\mu) T_{e}(E) d E\right\},
\end{aligned}
$$

where $\left\{-\left[\partial f_{e}(E, \mu, T) / \partial E\right]\right\}$ is a negative derivative of the Fermi function which effectively restricts the energy integration in a small region centered at the chemical potential as the temperature is finite. From Eq. (7a) it can be seen that $L_{0}$ is mainly determined by the electronic transmission coefficient $T_{e}(E)$ : the larger $T_{e}(E)$, the larger $L_{0}$. On the other hand, $L_{1}$ can be separated into two parts, for positive and negative values of $(E-\mu)$, respectively. By doing so we see that $L_{1}$ is proportional to the difference of $T_{e}(E)$ in the two parts. In Fig. 4(d), we report the electronic transmission coefficient $T_{e}$ of $A$-BCNNRs with width $W_{A}=5$ at $L_{B N}=L_{C}=6$ and $\mu=$ $1.58 \mathrm{eV}$ as a function of the electron energy. It is found that $T_{e}$ in the region $E<\mu$ decreases abruptly and an energy gap appears with increasing $N$, while $T_{e}$ at $E>\mu$ varies slowly, leading to $L_{0}$ decreasing and $L_{1}$ increasing. Accordingly, the Seebeck coefficient increases with the periodic number.

In Fig. 5, the influence of the length of $h$-BN and graphene on the thermoelectric properties of $A$-BCNNRs is studied. In the left column [(a), (c), and (e)], we fix the length of $h$-BN $L_{B N}$ and change $L_{C}$; while in the right column [(b), (d), and (f)] we fix $L_{C}$ and change $L_{B N}$. One can see that with the increase of the periodic number $N$, the figure of merit for nanoribbons with different widths gradually increases and 


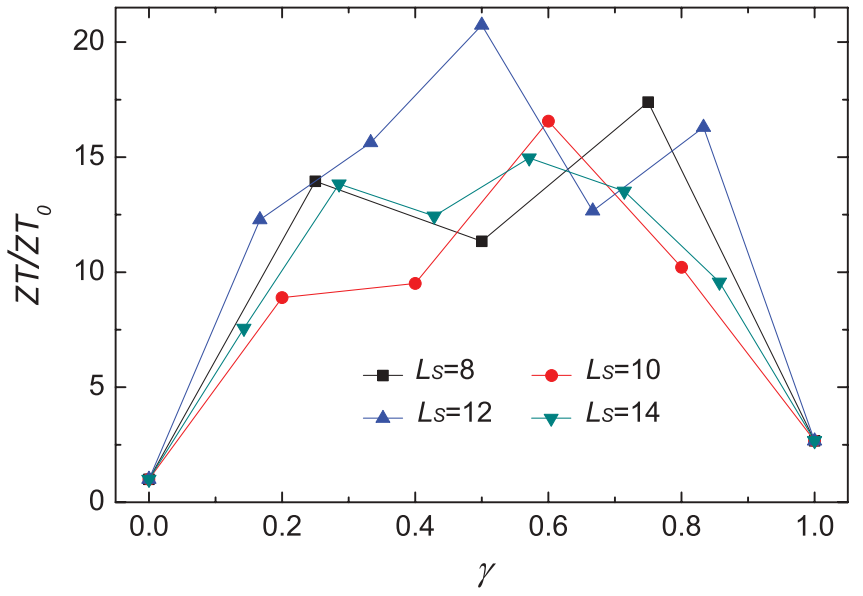

FIG. 6. (Color online) Thermoelectric figure of merit ratio $Z T / Z T_{0}$ of $A$-BCNNRs with width $W_{A}=5$ versus component ratio $\gamma$, at periodic number $N=10$ and temperature $T=300 \mathrm{~K}$.

finally trends to a constant. Compared to pristine $A$-GNRs, the $Z T$ of metallic nanoribbons is increased about 10-20 times [see the insets of Figs. 5(a) and 5(b)], while the $Z T$ of semiconducting nanoribbons is enhanced about 1.5-3 times [see the insets of Figs. 5(c)-5(f)]. From these results we can conclude that the thermoelectric properties of $A$-GNRs can be tuned by embedding $h$-BN.

We next investigate the effect of the component ratio of $h$-BN on the thermoelectric transport properties in $A$-BCNNRs. Figure 6 shows the figure of merit ratio $Z T / Z T_{0}$ versus $\gamma$ for nanoribbons with width $W_{A}=5$ under different supercell length $L_{S}$. One can find that $Z T / Z T_{0}$ at $\gamma=0$ and 1.0 is small, while in the range of $\gamma$ between 0.2 and 0.8 , in particular $\gamma$ near $0.5, Z T / Z T_{0}$ attains a relatively large value. The phenomenon can be qualitatively understood from the electronic energy band structure. For the pristine

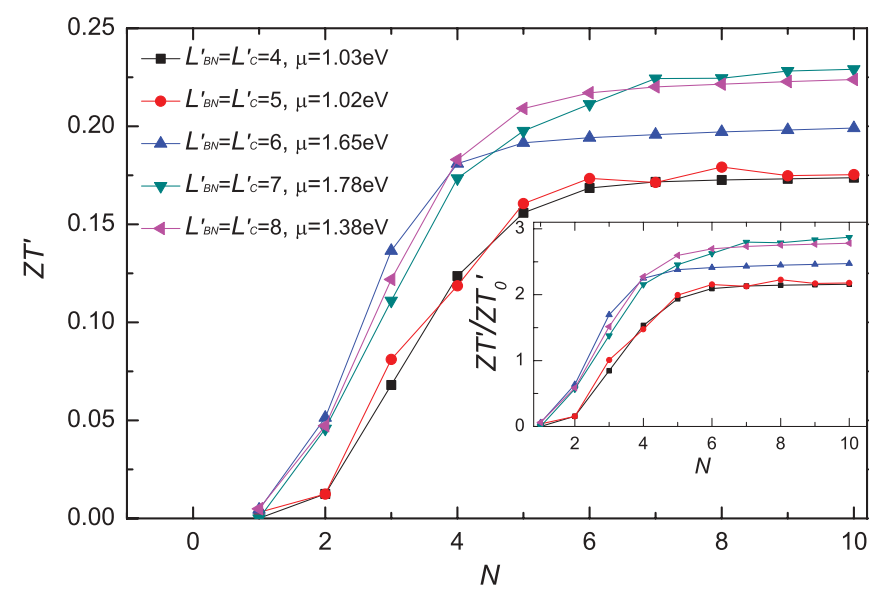

FIG. 7. (Color online) Thermoelectric figure of merit $Z T^{\prime}$ for $Z$-BCNNRs at room temperature, and the inset plots the ratio $Z T^{\prime} / Z T_{0}^{\prime}$ versus periodic number $N$, where $Z T_{0}^{\prime}$ is the result of pristine $Z$-GNRs. Here we take $\gamma^{\prime}=0.5\left(L_{B N}^{\prime}=L_{C}^{\prime}\right)$ and $W_{Z}=3$. For different lengths $L_{B N}^{\prime}$ and $L_{C}^{\prime}$, we take the chemical potential $(\mu)$ corresponding to the absolute maximum of the figure of merit.
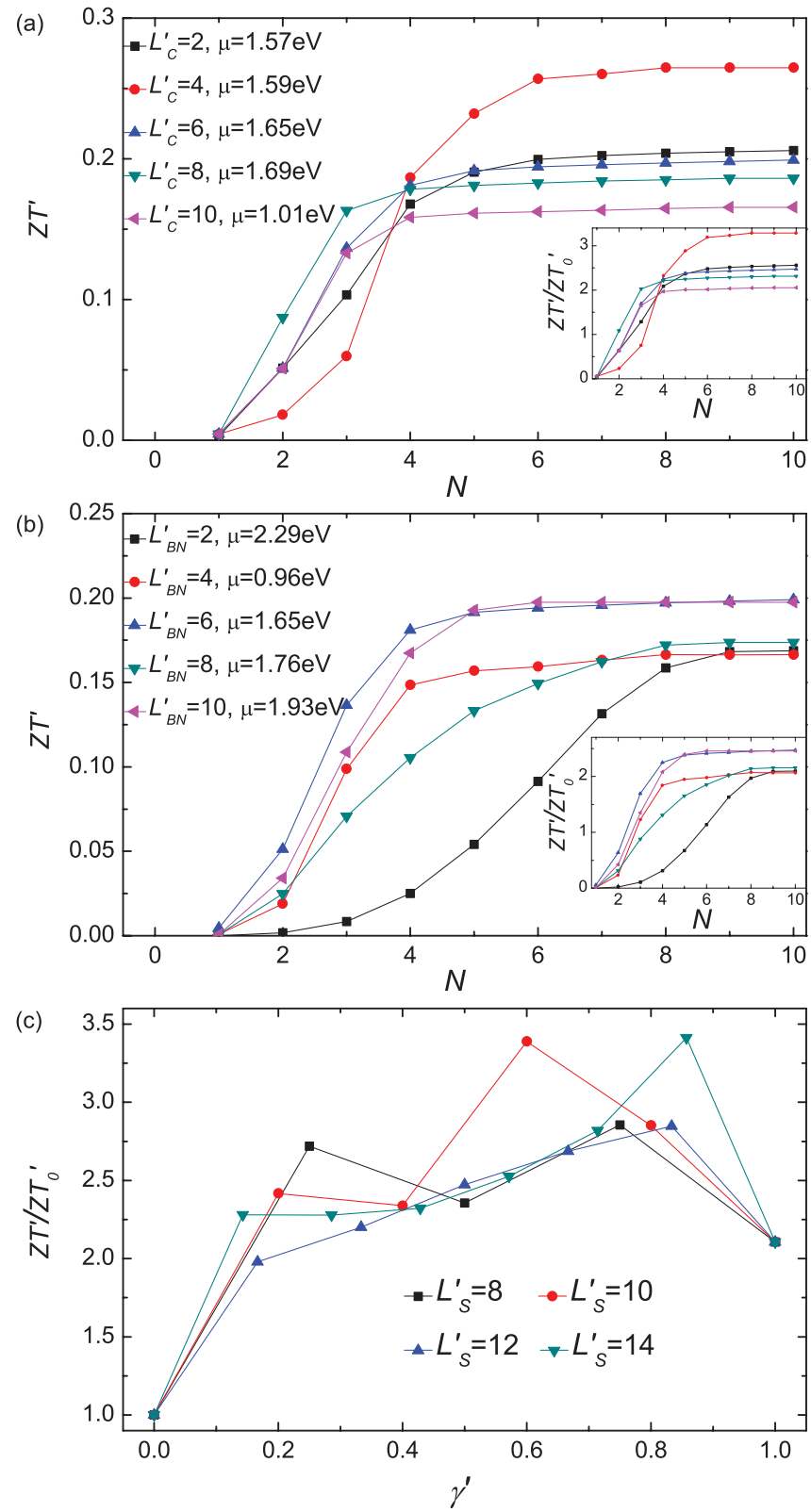

FIG. 8. (Color online) Thermoelectric figure of merit for $Z$-BCNNRs versus periodic number $N$ under various component ratio. In (a) $L_{B N}^{\prime}=6$, (b) $L_{C}^{\prime}=6$, and the insets represent the ratio $Z T^{\prime} / Z T_{0}^{\prime}$ of the nanoribbons. (c) Figure of merit ratio versus component ratio $\gamma^{\prime}$ for different supercell length $L_{S}^{\prime}$. We take the chemical potential $(\mu)$ in (a) and (b) corresponding to the absolute maximum of the figure of merit for given value $L_{B N}^{\prime}\left(L_{C}^{\prime}\right)$ and the other parameters are the same as in Fig. 7.

$A$-GNRs, there exist perfect channels of carrier transport. By periodically embedding $h$-BN into the $A$-GNRs, on the one hand, the transport channels are destroyed, resulting in the thermal conductance and electrical conductance reducing, while on the other hand, a number of minigaps appear in the transmission spectrum. When the value of the component ratio $\gamma$ gets close to 0.5 , the width of some minigaps is sufficient to contribute to a high Seebeck coefficient. Moreover, the high Seebeck coefficient and decreased thermal 
conductance outweigh the decreased electrical conductance. This implies that generating an effective electronic energy gap is important for improving the thermoelectric performance of materials.

The thermoelectric properties of $Z$-BCNNRs are discussed in Fig. 7 where we show the figure of merit $Z T^{\prime}$ as a function of the periodic number $N$ for zigzag-edged nanoribbons with width $W_{Z}=3$ at a fixed component ratio $\gamma^{\prime}=0.5$ $\left(L_{B N}^{\prime}=L_{C}^{\prime}\right)$, and the inset plots the ratio $Z T^{\prime} / Z T_{0}^{\prime}$, where $Z T_{0}^{\prime}$ corresponds to the figure of merit of $Z$-GNRs. One can find that likewise to the case of $A$-BCNNRs, $Z T^{\prime}$ gradually increases with the periodic number and ultimately reaches a constant value. The figure of merit $Z T^{\prime}$ for $Z$-BCNNRs is improved 2-3 times compared to that of perfect $Z$-GNRs, as shown in the inset. The enhancement of the figure of merit for zigzag-edged nanoribbons is smaller than that of the metallic $A$-GNRs, while it is slightly larger than that of the semiconducting $A$-GNRs as seen by comparing Fig. 7 with Fig. 2. The origin of this behavior can be attributed to the zigzag-edged nanoribbons with higher thermal conductance than that of the armchair-edged nanoribbons of comparable width. As to the semiconducting $A$-GNRs, because of the energy gap, the thermoelectric figure of merit $Z T_{0}$ is higher than $Z T_{0}^{\prime}$, which outweighs the discrepancy of thermal conductance, resulting in a $Z T / Z T_{0}$ falling.

Figure 8 investigates the thermoelectric properties of $Z$-BCNNRs under different component ratios of $h$-BN and graphene. We first fix the length of $h$-BN in the supercell and change the length of graphene [Fig. 8(a)], and then fix the length of graphene and change the length of $h$-BN [Fig. 8(b)]. The insets correspond to the figure of merit ratio $Z T^{\prime} / Z T_{0}^{\prime}$ as a function of periodic number $N$, where $Z T_{0}^{\prime}$ is the figure of merit of pristine $Z$-GNRs. One can find that the $Z T^{\prime}$ for $Z$-BCNNRs increases gradually with increasing the periodic number and finally reaches a constant. The enhanced magnitude, compared to the perfect $Z$-GNRs, is about 2-3 times (see the insets). The enhancement originates from the dramatically decreased thermal conductance and increased Seebeck coefficient outweighing the decreased electric conductance. In Fig. 8(c) the figure of merit ratio $Z T^{\prime} / Z T_{0}^{\prime}$ versus the component ratio $\gamma^{\prime}$ for various supercell lengths $L_{S}^{\prime}$ is studied. It can be seen that $Z T^{\prime} / Z T_{0}^{\prime}$ in the range of $\gamma^{\prime}$ from 0.2 to 0.9 exhibits quite larger values and even exceeds 3.0. When $\gamma^{\prime}$ grows towards $1.0, Z T^{\prime} / Z T_{0}^{\prime}$ decreases rapidly, while keeping a positive value in the tail because of the embedded $h$-BN.

\section{CONCLUSIONS}

In summary, we have investigated the thermoelectric transport properties of BCNNRs using the NEGF technique. It is found that the thermoelectric figure of merit can be significantly enhanced by periodically embedding $h$-BN into GNRs. Compared to the pristine GNRs, the $Z T$ of $A$-BCNNRs with the width index of $3 p+2$ is enhanced 10-20 times, while $Z T$ of nanoribbons with other widths is enhanced about 1.5-3 times; as to zigzag-edge, the figure of merit for BCNNRs is improved 2-3 times. The strong enhancement can be justified by the combined increase in the Seebeck coefficient and decrease in the thermal conductance outweighing the decrease in the electrical conductance. In addition, the effect of the component ratio of $h$-BN on the thermoelectric properties is discussed. It is shown that the figure of merit in the case of $\gamma\left(\gamma^{\prime}\right)$ nearing 0.5 exhibits a larger value. Our investigations demonstrate that the hybrid nanostructure composed of alternating $h$-BN and graphene is a very promising material for building highly efficient thermoelectric devices.

\section{ACKNOWLEDGMENTS}

We acknowledge financial support from Spanish MICINN (FIS2011-65702-C02-01 and PIB2010US-00652), ACI-Promociona (ACI2009-1036), Grupos Consolidados UPV/EHU del Gobierno Vasco (IT-319-07), Consolider nanoTHERM (Grant No. CSD2010-00044), the European Research Council Advanced Grant DYNamo (ERC-2010-AdG -Proposal No. 267374), and also the National Natural Science Foundation of China (Grants No. 51176161, No. 51006086, No. 11074213, and No. 10774127). *chenyp@xtu.edu.cn

†roberto.dagosta@ehu.es

‡angel.rubio@ehu.es

${ }^{1}$ A. I. Hochbaum, R. Chen, R. D. Delgado, W. Liang, E. C. Garnett, M. Najarian, A. Majumdar, P. Yang, Nature (London) 451, 163 (2008).

${ }^{2}$ X. Yan, G. Joshi, W. Liu, Y. Lan, H. Wang, S. Lee, J. W. Simonson, S. J. Poon, T. M. Tritt, G. Chen, and Z. F. Ren, Nano Lett. 11, 556 (2011).

${ }^{3}$ Y. M. Zuev, W. Chang, and P. Kim, Phys. Rev. Lett. 102, 096807 (2009).

${ }^{4}$ P. Wei, W. Bao, Y. Pu, C. N. Lau, and J. Shi, Phys. Rev. Lett. 102, 166808 (2009).

${ }^{5}$ L. Shi, D. Yao, G. Zhang, and B. Li, Appl. Phys. Lett. 95, 063102 (2009).

${ }^{6}$ F. Mazzamuto, V. Hung Nguyen, Y. Apertet, C. Caer, C. Chassat, J. Saint-Martin, and P. Dollfus, Phys. Rev. B 83, 235426 (2011).
${ }^{7}$ W. Huang, J. S. Wang, and G. Liang, Phys. Rev. B 84, 045410 (2011).

${ }^{8}$ L. Shi, D. Yao, G. Zhang, and B. Li, Appl. Phys. Lett. 95, 063102 (2009).

${ }^{9}$ X. Ni, G. Liang, J. S. Wang, and B. Li, Appl. Phys. Lett. 95, 192114 (2009).

${ }^{10}$ K. S. Novoselov, A. K. Geim, S. V. Morozov, D. Jiang, Y. Zhang, S. V. Dubonos, I. V. Grigorieva, and A. A. Firsov, Science 306, 666 (2004).

${ }^{11}$ K. S. Novoselov, D. Jiang, F. Schedin, T. J. Booth, V. V. Khotkevich, S. V. Morozov, and A. K. Geim, Proc. Natl. Acad. Sci. USA 102, 10451 (2005).

${ }^{12}$ L. Wirtz, A. Rubio, R. A. de la Concha, and A. Loiseau, Phys. Rev. B 68, 045425 (2003).

${ }^{13}$ A. Grüneis, C. Attaccalite, L. Wirtz, H. Shiozawa, R. Saito, T. Pichler, and A. Rubio, Phys. Rev. B 78, 205425 (2008). 
${ }^{14}$ L. Wirtz, A. Marini, M. Grüning, C. Attaccalite, G. Kresse, and A. Rubio, Phys. Rev. Lett. 100, 189701 (2008).

${ }^{15}$ J. Serrano, A. Bosak, R. Arenal, M. Krisch, K. Watanabe, T. Taniguchi, H. Kanda, A. Rubio, and L. Wirtz, Phys. Rev. Lett. 98, 095503 (2007).

${ }^{16}$ A. Grüneis, J. Serrano, A. Bosak, M. Lazzeri, S. L. Molodtsov, L. Wirtz, C. Attaccalite, M. Krisch, A. Rubio, F. Mauri, and T. Pichler, Phys. Rev. B 80, 085423 (2009).

${ }^{17}$ A. Rubio, J. L. Corkill, and M. L. Cohen, Phys. Rev. B 49, 5081 (1994).

${ }^{18}$ X. Blase, A. Rubio, S. G. Louie, and M. L. Cohen, Europhys. Lett. 28, 335 (1994).

${ }^{19}$ E. H. Hwang, S. Adam, and S. Das Sarma, Phys. Rev. Lett. 98, 186806 (2007).

${ }^{20}$ Y. Xing, Q. F. Sun, and J. Wang, Phys. Rev. B 80, 235411 (2009).

${ }^{21}$ Y. Ouyang and J. Guo, Appl. Phys. Lett. 94, 263107 (2009).

${ }^{22}$ A. A. Balandin, S. Ghosh, W. Z. Bao, I. Calizo, D. Teweldebrhan, F. Miao, and C. N. Lau, Nano Lett. 8, 902 (2008).

${ }^{23}$ S. Ghosh, I. Calizo, D. Teweldebrhan, E. P. Pokatilov, D. L. Nika, A. A. Balandin, W. Bao, F. Miao, and C. N. Lau, Appl. Phys. Lett. 92, 151911 (2008).

${ }^{24}$ T. Ouyang, Y. P. Chen, Y. E. Xie, K. K. Yang, Z. G. Bao, and J. X. Zhong, Nanotechnology 21, 245701 (2010).

${ }^{25}$ K. K. Yang, Y. P. Chen, Y. E. Xie, X. L. Wei, T. Ouyang, and J. X. Zhong, Solid State Commun. 151, 460 (2011).

${ }^{26}$ L. Ci, L. Song, C. Jin, D. Jariwala, D. Wu, Y. Li, A. Srivastava, Z. F. Wang, K. Storr, L. Balicas, F. Liu, and P. M. Ajayan, Nature Mater. 9, 430 (2010).

${ }^{27}$ A. Y. Liu, R. M. Wentzcovitch, and M. L. Cohen, Phys. Rev. B 39, 1760 (1989).

${ }^{28}$ O. Stephan, P. M. Ajayan, C. Colliex, Ph. Redlich, J. M. Lambert, P. Bernier, and P. Lefin, Science 266, 1683 (1994).

${ }^{29}$ H. Nozaki and S. Itoh, J. Phys. Chem. Solids 57, 41 (1996).

${ }^{30}$ X. Blase, J.-Ch. Charlier, A. De Vita, and R. Car, Appl. Phys. Lett. 70, 197 (1997); Appl. Phys. A: Mater. Sci. Process. 68, 293 (1999).

${ }^{31}$ M. S. C. Mazzoni, R. W. Nunes, S. Azevedo, and H. Chacham, Phys. Rev. B 73, 073108 (2006).

${ }^{32}$ J. da Rocha Martins and H. Chacham, ACS Nano 5, 385 (2011).

${ }^{33}$ P. Ayala, R. Arenal, A. Loiseau, A. Rubio, and T. Pichler, Rev. Mod. Phys. 82, 1843 (2010).

${ }^{34} B-C-N$ Nanotubes and Related Nanostructures, edited by Y. K. Yap, Lecture Notes in Nanoscale Science and Technology Vol. 6 (Springer, New York, 2009).
${ }^{35}$ Y. Miyamoto, A. Rubio, M. L. Cohen, and S. G. Louie, Phys. Rev. B 50, 4976 (1994).

${ }^{36}$ D. Tománek, R. M. Wentzcovitch, S. G. Louie, and M. L. Cohen, Phys. Rev. B 37, 3134 (1988).

${ }^{37}$ R. Ma, D. Golberg, Y. Bando, and T. Sasaki, Philos. Trans. R. Soc. Lond., Ser. A 362, 2161 (2004).

${ }^{38}$ Z. Weng-Sieh, K. Cherrey, N. G. Chopra, X. Blase, Y. Miyamoto, A. Rubio, M. L. Cohen, S. G. Louie, A. Zettl, and R. Gronsky, Phys. Rev. B 51, 11229 (1995).

${ }^{39}$ A. Rubio, Nature Mater. 9, 379 (2010).

${ }^{40}$ L. Song, L. Balicas, D. J. Mowbray, R. B. Capaz, K. Storr, L. Ci, D. Jariwala, S. Kurth, S. G. Louie, A. Rubio, and P. M. Ajayan, arXiv:1105.1876v2.

${ }^{41}$ K. T. Lam, Y. Lu, Y. P. Feng, and G. Liang, Appl. Phys. Lett. 98, 022101 (2011)

${ }^{42}$ Y. Ding, Y. Wang, and J. Ni, Appl. Phys. Lett. 95, 123105 (2009).

${ }^{43}$ G. Seol and J. Guo, Appl. Phys. Lett. 98, 143107 (2011).

${ }^{44}$ E. J. Kan, X. Wu, Z. Li, X. C. Zeng, J. Yang, and J. G. Hou, J. Chem. Phys. 129, 084712 (2008).

${ }^{45}$ J. W. Jiang, J. S. Wang, and B. S. Wang, Appl. Phys. Lett. 99, 043109 (2011)

${ }^{46}$ D. G. Cahill, W. K. Ford, K. E. Goodson, G. D. Mahan, A. Majumdar, H. J. Maris, R. Merlin, and S. R. Phillpot, J. Appl. Phys. 93, 793 (2003).

${ }^{47}$ S. Datta, Electronic Transport in Mesoscopic Systems (Cambridge University Press, Cambridge, 1995).

${ }^{48}$ T. Yamamoto and K. Watanabe, Phys. Rev. Lett. 96, 255503 (2006).

${ }^{49}$ J. S. Wang, J. Wang, and J. T. Lü, Eur. Phys. J. B 62, 381 (2008).

${ }^{50}$ K. K. Yang, Y. P. Chen, Y. E. Xie, T. Ouyang, and J. X. Zhong, Europhys. Lett. 91, 46006 (2010).

${ }^{51}$ N. Mingo, Phys. Rev. B 74, 125402 (2006).

${ }^{52}$ J. T. Lü and J. S. Wang, Phys. Rev. B 76, 165418 (2007).

${ }^{53}$ T. C. Li and S. P. Lu, Phys. Rev. B 77, 085408 (2008).

${ }^{54}$ F. Chen, Y. P. Chen, M. Zhang, and J. X. Zhong, Chin. Phys. B 19, 068105 (2010).

${ }^{55}$ M. P. L. Sancho, J. M. L. Sancho, and J. Rubio, J. Phys. F: Met. Phys. 15, 851 (1985).

${ }^{56}$ L. Wirtz and A. Rubio, Solid State Commun. 131, 141 (2004).

${ }^{57}$ R. Saito, G. Dresselhaus, and M. Dresselhaus, Physical Properties of Carbon Nanotubes (Imperial College Press, London, 1998).

${ }^{58}$ Y. Xiao, X. H. Yan, J. X. Cao, J. W. Ding, Y. L. Mao, and J. Xiang, Phys. Rev. B 69, 205415 (2004). 